\title{
Explicit formulas and global uniqueness for phaseless inverse scattering in multidimensions
}

\section{R.G. Novikov}

CNRS (UMR 7641), Centre de Mathématiques Appliquées, Ecole Polytechnique, 91128 Palaiseau, France;

IEPT RAS, 117997 Moscow, Russia

e-mail: novikov@cmap.polytechnique.fr

Abstract. We consider phaseless inverse scattering for the Schrödinger equation with compactly supported potential in dimension $d \geq 2$. We give explicit formulas for solving this problem from appropriate data at high energies. As a corollary, we give also a global uniqueness result for this problem with appropriate data on a fixed energy neighborhood.

\section{Introduction}

We consider the Schrödinger equation

$$
-\Delta \psi+v(x) \psi=E \psi, \quad x \in \mathbb{R}^{d}, d \geq 2, E>0,
$$

where

$$
\begin{aligned}
& v \in L^{\infty}\left(\mathbb{R}^{d}\right), \quad \text { supp } v \subset D, \\
& D \text { is an open bounded domain in } \mathbb{R}^{d} .
\end{aligned}
$$

For equation (1.1) we consider the classical scattering solutions $\psi^{+}=\psi^{+}(x, k), x \in \mathbb{R}^{d}$, $k \in \mathbb{R}^{d}, k^{2}=E$, specified by the following asymptotics as $|x| \rightarrow \infty$ :

$$
\begin{aligned}
& \psi^{+}(x, k)=e^{i k x}+c(d,|k|) \frac{e^{i|k||x|}}{|x|^{(d-1) / 2}} f\left(k,|k| \frac{x}{|x|}\right)+O\left(\frac{1}{|x|^{(d+1) / 2}}\right), \\
& c(d,|k|)=-\pi i(-2 \pi i)^{(d-1) / 2}|k|^{(d-3) / 2}
\end{aligned}
$$

for some a priori unknown $f$. In addition, the function $f=f(k, l), k, l \in \mathbb{R}^{d}, k^{2}=l^{2}=E$, arising in (1.3) is the classical scattering amplitude for equation (1.1).

In order to find $\psi^{+}$and $f$ from $v$ one can use, in particular, the Lippmann-Schwinger integral equation

$$
\begin{aligned}
& \psi^{+}(x, k)=e^{i k x}+\int_{D} G^{+}(x-y, k) v(y) \psi^{+}(y, k) d y \\
& G^{+}(x, k)=-(2 \pi)^{-d} \int_{\mathbb{R}^{d}} \frac{e^{i \xi x} d \xi}{\xi^{2}-k^{2}-i 0}
\end{aligned}
$$

and the formula

$$
f(k, l)=(2 \pi)^{-d} \int_{D} e^{-i l y} v(y) \psi^{+}(y, k) d y
$$


R.G. Novikov

where $x, k, l \in \mathbb{R}^{d}, k^{2}=l^{2}=E>0$; see e.g. [BS], [F2].

The scattering amplitude $f$ for equation (1.1) at fixed $E$ is defined on

$$
\mathcal{M}_{E}=\left\{k \in \mathbb{R}^{d}, l \in \mathbb{R}^{d}: k^{2}=l^{2}=E\right\}, \quad E>0 .
$$

In addition to $f$ on $\mathcal{M}_{E}$, we consider also $\left.f\right|_{\Gamma_{E}}$, where

$$
\begin{aligned}
& \Gamma_{E}=\left\{k=k_{E}(p), l=l_{E}(p): p \in \overline{\mathcal{B}}_{2 \sqrt{E}}\right\}, \\
& k_{E}(p)=\frac{p}{2}+\left(E-\frac{p^{2}}{4}\right)^{1 / 2} \gamma(p), l_{E}(p)=-\frac{p}{2}+\left(E-\frac{p^{2}}{4}\right)^{1 / 2} \gamma(p), \\
& \mathcal{B}_{r}=\left\{p \in \mathbb{R}^{d}:|p|<r\right\}, \quad \overline{\mathcal{B}}_{r}=\left\{p \in \mathbb{R}^{d}:|p| \leq r\right\}, \quad r>0,
\end{aligned}
$$

where $\gamma$ is a piecewise continuous vector-function on $\mathbb{R}^{d}$ such that

$$
|\gamma(p)|=1, \quad \gamma(p) p=0, \quad p \in \mathbb{R}^{d} .
$$

One can see that

$$
\Gamma_{E} \subset \mathcal{M}_{E}, \operatorname{dim} \Gamma_{E}=d, \quad \operatorname{dim} \mathcal{M}_{E}=2 d-2, E>0, d \geq 2 .
$$

Let

$$
\mathcal{M}_{\Lambda}=\cup_{E \in \Lambda} \mathcal{M}_{E}, \quad \Gamma_{\Lambda}=\cup_{E \in \Lambda} \Gamma_{E}
$$

where $\left.\Lambda \subseteq \mathbb{R}_{+}=\right] 0,+\infty[$.

We start with the following inverse scattering problems for equation (1.1) under assumptions (1.2):

Problem 1.1. Reconstruct potential $v$ on $\mathbb{R}^{d}$ from its scattering amplitude $f$ on some appropriate $\mathcal{M}^{\prime} \subseteq \mathcal{M}_{\mathbb{R}_{+}}$.

Problem 1.2. Reconstruct potential $v$ on $\mathbb{R}^{d}$ from its phaseless scattering data $|f|^{2}$ on some appropriate $\mathcal{M}^{\prime} \subseteq \mathcal{M}_{\mathbb{R}_{+}}$.

Note that in quantum mechanical scattering experiments (in framework of model described by equation (1.1)) the phaseless scattering data $|f|^{2}$ can be measured directly, whereas the complete scattering amplitude $f$ is not accessible for direct measurements. Therefore, Problem 1.2 is of particular interest from applied point of view in the framework of quantum mechanical inverse scattering. However, in the literature much more results are given on Problem 1.1 (see [ABR],[B], [BAR], [ChS], [EW], [E], [ER], [F1], [F2], [G], [HH], $[\mathrm{HN}],[\mathrm{I}],[\mathrm{IN}],[\mathrm{Me}],[\mathrm{Mo}],[\mathrm{Ne}],[\mathrm{N} 1]-[\mathrm{N} 7],[\mathrm{R}],[\mathrm{S}],[\mathrm{VW}],[\mathrm{WY}]$ and references therein) than on Problem 1.2 (see chapter $\mathrm{X}$ of [ChS] and recent works [K1], [K2] and references therein, where in [K1], [K2] some similar problem is considered).

In particular, for Problem 1.1 it is well known that the scattering amplitude $f$ at high energies uniquely determines $v$ via the formulas

$$
\begin{aligned}
& \hat{v}(k-l)=f(k, l)+O\left(E^{-1 / 2}\right) \text { as } E \rightarrow+\infty, \quad(k, l) \in \mathcal{M}_{E}, \\
& \hat{v}(p)=(2 \pi)^{-d} \int_{D} e^{i p x} v(x) d x, \quad p \in \mathbb{R}^{d}
\end{aligned}
$$


Explicit formulas and global uniqueness for phaseless inverse scattering in multidimensions

see, for example, [F1], [N7].

On the other hand, for Problem 1.2 it is well known that the phaseless scattering data $|f|^{2}$ on $\mathcal{M}_{\mathbb{R}_{+}}$do not determine $v$ uniquely, in general. In particular, we have that

$$
\begin{aligned}
& f_{y}(k, l)=e^{i(k-l) y} f(k, l), \\
& \left|f_{y}(k, l)\right|^{2}=|f(k, l)|^{2}, \quad(k, l) \in \mathcal{M}_{\mathbb{R}_{+}}, y \in \mathbb{R}^{d}
\end{aligned}
$$

where $f$ is the scattering amplitude for $v$ and $f_{y}$ is the scattering amplitude for $v_{y}$, where

$$
v_{y}(x)=v(x-y), \quad x \in \mathbb{R}^{d}, \quad y \in \mathbb{R}^{d}
$$

see, for example, Lemma 1 of [N6].

In the present work, in view of the aforementioned nonuniqueness for Problem 1.2 we modify this problem into Problem 1.3 formulated below. Let

$$
S=\left\{|f|^{2},\left|f_{j}\right|^{2}, \quad j=1, \ldots, n\right\}
$$

where $f$ is the initial scattering amplitude for $v$ satisfying (1.2) and $f_{j}$ is the scattering amplitude for

$$
v_{j}=v+w_{j}, \quad j=1, \ldots, n,
$$

where $w_{1}, \ldots, w_{n}$ are additional a priori known background scatterers such that

$$
\begin{aligned}
& w_{j} \in L^{\infty}\left(\mathbb{R}^{d}\right), \text { supp } w_{j} \subset \Omega_{j}, \\
& \Omega_{j} \text { is an open bounded domain in } \mathbb{R}^{d}, \Omega_{j} \cap D=\emptyset, \\
& \quad w_{j} \neq 0, w_{j_{1}} \neq w_{j_{2}} \text { for } j_{1} \neq j_{2} \text { in } L^{\infty}\left(\mathbb{R}^{d}\right), \\
& \quad j, j_{1}, j_{2} \in\{1, \ldots, n\} .
\end{aligned}
$$

Problem 1.3. Reconstruct potential $v$ on $\mathbb{R}^{d}$ from the phaseless scattering data $S$ on some appropriate $\mathcal{M}^{\prime} \subseteq \mathcal{M}_{\mathbb{R}_{+}}$and for some appropriate background scatterers $w_{1}, \ldots, w_{n}$.

Note also that Problems 1.1, 1.2, 1.3 can be considered as examples of ill-posed problems; see [LRS] for an introduction to this theory.

Problem 1.3 in dimension $d=1$ was, actually, considered in [AS] for $n=1$. However, to our knowledge, Problem 1.3 in dimension $d \geq 2$ was not yet considered in the literature before the present work.

Results of the present work can be summarized as follows.

First, we give explicit formulas for solving Problem 1.3 in dimension $d \geq 2$ for $n=2$ and $\mathcal{M}^{\prime}=\Gamma_{\Lambda}$ defined by (1.7), (1.11) for any unbounded $\Lambda \subset \mathbb{R}_{+}$; see Theorem 2.1, Remark 3.1 and Corollary 2.1 of Section 2. As an example of $\Lambda$ for this result one can take $\Lambda=\left[E_{0},+\infty\left[, E_{0}>0\right.\right.$, or just $\Lambda$ of Remark 2.1 .

Second, we give a global uniqueness result for Problem 1.3 in dimension $d \geq 2$ for $n=2$ and $\mathcal{M}^{\prime}=\Gamma_{\Lambda}$ for any bounded infinite $\Lambda \subset \mathbb{R}_{+}$; see Theorem 2.2 of Section 2 . As an 
example of $\Lambda$ for this result one can take $\Lambda=] E_{0}-\varepsilon, E_{0}+\varepsilon\left[, E_{0}>0, \varepsilon>0, E_{0}-\varepsilon \geq 0\right.$, or just $\Lambda$ of Theorem 2.2.

In addition, we indicate possible extensions of the aforementioned results to the case $n=1$; see Propositions 2.1, 2.2 of Section 2 .

The progress of the present work in comparison with the recent works [K1], [K2] includes explicit formulas for phaseless inverse scattering at high energies and no assumption that $v \geq 0$. In addition, in the present work we consider inverse scattering from far field phaseless scattering data (and not from near field phaseless scattering data as in [K1], $[\mathrm{K} 2])$.

The main statements of the present work are presented in detail in the next section.

\section{Main statements}

2.1. Notations and related remarks. Let

$$
\hat{u}(p)=(2 \pi)^{-d} \int_{\mathbb{R}^{d}} e^{i p x} u(x) d x, \quad p \in \mathbb{R}^{d},
$$

where $u$ is a test function on $\mathbb{R}^{d}$. In particular, we consider $\hat{u}=\hat{v}, \hat{w}_{j}$ for $u=v, w_{j}$, $j=1, \ldots, n$, where $v, w_{j}$ satisfy (1.2), (1.18).

Note that if

$$
u_{y}(x)=u(x-y), \quad x, y \in \mathbb{R}^{d},
$$

then

$$
\hat{u}_{y}(p)=e^{i p y} \hat{u}(p), \quad p \in \mathbb{R}^{d} .
$$

We represent $\hat{v}$ and $\hat{w}_{j}$ as follows:

$$
\begin{aligned}
& \hat{v}(p)=|\hat{v}(p)| \theta(p), \quad \theta(p)=e^{i \alpha(p)}, \\
& \hat{w}_{j}(p)=\left|\hat{w}_{j}(p)\right| \omega_{j}(p), \quad \omega_{j}(p)=e^{i \beta_{j}(p)},
\end{aligned}
$$

where $p \in \mathbb{R}^{d}, j=1, \ldots, n$.

We consider the following sets:

$$
\begin{aligned}
& A_{y}=\left\{p \in \mathbb{R}^{d}: \quad e^{2 i p y}=1\right\}, \quad y \in \mathbb{R}^{d} \\
& Z_{0}=\left\{p \in \mathbb{R}^{d}: \quad|\hat{v}(p)|=0\right\}, Z_{j}=\left\{p \in \mathbb{R}^{d}: \quad\left|\hat{w}_{j}(p)\right|=0\right\}, \quad j=1, \ldots, n, \\
& Y_{j_{1}, j_{2}}=\left\{p \in \mathbb{R}^{d} \backslash\left(Z_{j_{1}} \cup Z_{j_{2}}\right):\left(\omega_{j_{1}}(p)\right)^{2}=\left(\omega_{j_{2}}(p)\right)^{2}\right\}, \quad 1 \leq j_{1}, j_{2} \leq n, j_{1} \neq j_{2} .
\end{aligned}
$$

We have, in particular, that

$$
A_{y} \text { is closed and } M e s A_{y}=0 \text { in } \mathbb{R}^{d}, y \neq 0 \text {. }
$$

Assumptions (1.2) on $v$ imply, in particular, that $\hat{v}$ is (complex-valued) real-analytic on $\mathbb{R}^{d}$. Therefore:

$$
Z_{0} \text { is closed in } \mathbb{R}^{d} ; \text { Mes } Z_{0}=0 \text { in } \mathbb{R}^{d} \text { if } \hat{v} \not \equiv 0 \text {. }
$$


Explicit formulas and global uniqueness for phaseless inverse scattering in multidimensions

Assumptions (1.18) on $w_{j}$ imply, in particular, that $\hat{w}_{j}$ is (complex-valued) realanalytic on $\mathbb{R}^{d}$ and $\hat{w}_{j} \not \equiv 0, j=1, \ldots, n$. Therefore,

$$
Z_{j} \text { is closed and } M e s Z_{j}=0 \text { in } \mathbb{R}^{d}, j=1, \ldots, n \text {. }
$$

In addition, if

$$
w_{j}(x)=w_{j}^{0}(|x-y|), \quad x \in \mathbb{R}^{d}, \text { for some } w_{j}^{0},
$$

for some $j$ and some $y \in \mathbb{R}^{d}$, then

$$
Z_{j}=\left\{p \in \mathbb{R}^{d}:|p| \in \mathcal{R}_{j}\right\}
$$

where $\mathcal{R}_{j}$ is a discrete set in $\mathbb{R}_{+}$without accumulation points (except $+\infty$ ) and $\mathcal{R}_{j}$ is independent of $y$.

In addition, taking into account (2.2), if

$$
w_{j_{2}}(x)=w_{j_{1}}(x-y), \quad x \in \mathbb{R}^{d}, \quad j_{2} \neq j_{1},
$$

for some $j_{1}, j_{2}$ and some $y \in \mathbb{R}^{d} \backslash\{0\}$, then

$$
Y_{j_{1}, j_{2}} \subseteq A_{y}
$$

2.2. Results on Problem 1.3 in dimension $d \geq 2$ for $n=2$.

Theorem 2.1. Suppose that complex-valued $v$ satisfies (1.2), complex-valued $w_{j}$ satisfies (1.18a), $j=1,2, d \geq 2$. Then the following formulas hold:

$$
\begin{gathered}
\left|\hat{v}_{j}(p)\right|^{2}=\lim _{\substack{p=k-l,(k, l) \in \mathcal{M}_{E} \\
E \rightarrow+\infty}}\left|f_{j}(k, l)\right|^{2} \text { for each } p \in \mathbb{R}^{d}, j=0,1,2, \\
\left.|| \hat{v}_{j}(p)\right|^{2}-\left|f_{j}(k, l)\right|^{2} \mid \leq c\left(D_{j}\right) N_{j}^{3} E^{-1 / 2}, \\
p=k-l, \quad(k, l) \in \mathcal{M}_{E}, E^{1 / 2} \geq \rho\left(D_{j}, N_{j}\right), j=0,1,2,
\end{gathered}
$$

where $v_{0}=v, f_{0}=f, D_{0}=D, v_{j}$ is defined by (1.17) and $D_{j}=D \cup \Omega_{j}$ for $j=1,2$, $\left\|v_{j}\right\|_{L^{\infty}\left(D_{j}\right)} \leq N_{j}, j=0,1,2$, and $c, \rho$ are defined by (3.10), (3.11). Suppose, in addition, that $w_{1}, w_{2}$ satisfy $(1.18 b)$ and that

$$
\operatorname{Mes} \bar{Y}_{1,2}=0 \text { in } \mathbb{R}^{d}
$$

where $\bar{Y}_{1,2}$ denotes the closure of $Y_{1,2}$ in $\mathbb{R}^{d}$. Then the following formula holds:

$$
\begin{aligned}
& \left(\begin{array}{c}
\cos \alpha \\
\sin \alpha
\end{array}\right)=\left(\sin \left(\beta_{2}-\beta_{1}\right)\right)^{-1} \times \\
& \left(\begin{array}{cc}
\sin \beta_{2} & -\sin \beta_{1} \\
-\cos \beta_{2} & \cos \beta_{1}
\end{array}\right)\left(\begin{array}{c}
\left(2|\hat{v}|\left|\hat{w}_{1}\right|\right)^{-1}\left(\left|\hat{v}_{1}\right|^{2}-|\hat{v}|^{2}-\left|\hat{w}_{1}\right|^{2}\right) \\
\left(2|\hat{v}|\left|\hat{w}_{2}\right|\right)^{-1}\left(\left|\hat{v}_{2}\right|^{2}-|\hat{v}|^{2}-\left|\hat{w}_{2}\right|^{2}\right)
\end{array}\right),
\end{aligned}
$$


$\alpha=\alpha(p),|\hat{v}|=|\hat{v}(p)|, \beta_{j}=\beta_{j}(p),\left|\hat{w}_{j}\right|=\left|\hat{w}_{j}(p)\right|, j=1,2, p \in \mathbb{R}^{d} \backslash\left(Z_{0} \cup Z_{1} \cup Z_{2} \cup \bar{Y}_{1,2}\right)$, where $\alpha, \beta_{1}, \beta_{2}$ are defined in (2.3).

Theorem 2.1 is proved in Section 3 .

Remark 2.1. Formulas (2.14), (2.15) of Theorem 2.1 remain valid with $\Gamma_{E}$ in place of $\mathcal{M}_{E}$, where $\Gamma_{E}$ is defined by (1.7). In addition, taking into account (2.9), (2.16) these formulas can be considered as explicit formulas for finding $\hat{v}$ on $\mathbb{R}^{d}$ from $S=\left\{|f|^{2},\left|f_{1}\right|^{2},\left|f_{2}\right|^{2}\right\}$ on $\Gamma_{\Lambda}$ and background $w_{1}, w_{2}$ for any

$$
\Lambda=\left\{E_{j} \in \mathbb{R}_{+}: \quad j \in \mathbb{N}, \quad E_{j} \rightarrow \infty \text { as } j \rightarrow \infty\right\}
$$

where $\Gamma_{\Lambda}$ is defined in (1.11).

Corollary 2.1. Let all assumptions of Theorem 2.1 on $v$ and $w_{1}, w_{2}$ be fulfilled. Let $\Lambda$ be defined as in (2.18). Then $S=\left\{|f|^{2},\left|f_{1}\right|^{2},\left|f_{2}\right|^{2}\right\}$ on $\Gamma_{\Lambda}$ and background $w_{1}$, $w_{2}$ uniquely determine $v$ in $L^{\infty}\left(\mathbb{R}^{d}\right)$ via formulas $(2.14)$, (2.15) and the inverse Fourier transform.

In addition to results of Theorem 2.1, Remark 2.1 and Corollary 2.1 on the explicit reconstruction from phaseless scattering data at high energies, we have also the following global uniqueness result for the case of finite energies:

Theorem 2.2. Let $v$ satisfy (1.2), $w_{1}, w_{2}$ satisfy (1.18), (2.16), $d \geq 2$, and $v, w_{1}, w_{2}$ be real-valued. Let

$$
\Lambda=\left\{E_{j} \in \mathbb{R}_{+}: j \in \mathbb{N}, \quad E_{j_{1}} \neq E_{j_{2}} \text { for } j_{1} \neq j_{2}, E_{j} \rightarrow E_{*} \text { as } j \rightarrow \infty\right\}, \quad E_{*}>0
$$

Then $S=\left\{|f|^{2},\left|f_{1}\right|^{2},\left|f_{2}\right|^{2}\right\}$ on $\Gamma_{\Lambda}$ and background $w_{1}$, w $w_{2}$ uniquely determine $v$ in $L^{\infty}\left(\mathbb{R}^{d}\right)$.

Theorem 2.2 is proved in Section 4.

2.3. Results for the case $n=1$.

Proposition 2.1. If complex-valued $v$ satisfies (1.2), complex-valued $w_{1}$ satisfies (1.18a), $d \geq 2$, then formulas (2.14), (2.15) hold for $j=0,1$. If, in addition, $w_{1} \neq 0$ in $L^{\infty}\left(\mathbb{R}^{d}\right)$, then

$$
\begin{aligned}
& \cos \left(\alpha-\beta_{1}\right)=\left(2|\hat{v}|\left|\hat{w}_{1}\right|\right)^{-1}\left(\left|\hat{v}_{1}\right|^{2}-|\hat{v}|^{2}-\left|\hat{w}_{1}\right|^{2}\right), \\
& \alpha=\alpha(p),|\hat{v}|=|\hat{v}(p)|, \beta_{1}=\beta_{1}(p),\left|\hat{w}_{1}\right|=\left|\hat{w}_{1}(p)\right|, p \in \mathbb{R}^{d} \backslash\left(Z_{0} \cup Z_{1}\right),
\end{aligned}
$$

where $\alpha, \beta_{1}$ are defined in (2.3).

Proposition 2.1 is proved in Section 3.

Proposition 2.2. (A) There are not more than two different complex-valued potentials $v$ satisfying (1.2) with given $S=\left\{|f|^{2},\left|f_{1}\right|^{2}\right\}$ on $\Gamma_{\Lambda}$ and background complex-valued $w_{1}$ satisfying (1.18a), $w_{1} \neq 0$ in $L^{\infty}\left(\mathbb{R}^{d}\right)$, where $\Lambda$ is defined as in (2.18). (B) There are not more than two different real-valued potentials $v$ satisfying (1.2) with given $S=\left\{|f|^{2},\left|f_{1}\right|^{2}\right\}$ on $\Gamma_{\Lambda}$ and background real-valued $w_{1}$ satisfying $(1.18 \mathrm{a}), w_{1} \neq 0$ in $L^{\infty}\left(\mathbb{R}^{d}\right)$, where $\Lambda$ is defined as in (2.19). 
Explicit formulas and global uniqueness for phaseless inverse scattering in multidimensions

Proposition 2.2 is proved in Section 5 .

\section{Proofs of Proposition 2.1 and Theorem 2.1}

3.1. Preliminaries. Let

$$
\begin{aligned}
& L_{\sigma}^{\infty}\left(\mathbb{R}^{d}\right)=\left\{u \in L^{\infty}\left(\mathbb{R}^{d}\right):\|u\|_{\sigma}<+\infty\right\}, \\
& \|u\|_{\sigma}=\text { ess } \sup _{x \in \mathbb{R}^{d}}\left(1+|x|^{2}\right)^{\sigma / 2}|u(x)|, \quad \sigma \geq 0 .
\end{aligned}
$$

Note that

$$
v, w_{j}, v_{j} \in L_{\sigma}^{\infty}\left(\mathbb{R}^{d}\right) \text { for each } \sigma \geq 0,
$$

where $v, w_{j}, v_{j}, j \in\{1,2\}$, are the potentials of Proposition 2.1 and Theorem 2.1.

We recall that

$$
\begin{aligned}
& \left\|<x>^{-s} G^{+}(k)<x>^{-s}\right\|_{L^{2}\left(\mathbb{R}^{d}\right) \rightarrow L^{2}\left(\mathbb{R}^{d}\right)} \leq a_{0}(d, s)|k|^{-1}, \\
& k \in \mathbb{R}^{d}, \quad|k| \geq 1, \text { for } s>1 / 2
\end{aligned}
$$

where $G^{+}(k)$ denotes the integral operator with the Schwartz kernel $G^{+}(x-y, k)$ of $(1.4)$, $\langle x\rangle$ denotes the multiplication operator by the function $\left(1+|x|^{2}\right)^{1 / 2}$; see $[\mathrm{E}]$, [J] and references therein.

We will use the following detailed version of formula (1.12):

$$
\begin{aligned}
& |f(k, l)-\hat{v}(k-l)| \leq 2(2 \pi)^{-d} a_{0}(d, \sigma / 2)\left(c_{1}(d, \sigma)\|v\|_{\sigma}\right)^{2} E^{-1 / 2} \\
& (k, l) \in \mathcal{M}_{E}, \quad E^{1 / 2} \geq \rho_{1}\left(d, \sigma,\|v\|_{\sigma}\right), \sigma>d
\end{aligned}
$$

where $a_{0}(d, s)$ is the constant of $(3.3)$,

$$
\begin{aligned}
& c_{1}(d, \sigma)=\left(\int_{\mathbb{R}^{d}} \frac{d x}{\left(1+|x|^{2}\right)^{\sigma / 2}}\right)^{1 / 2}, \\
& \rho_{1}(d, \sigma, R)=\max \left(2 a_{0}(d, \sigma / 2) R, 1\right) ;
\end{aligned}
$$

see formula $(2.11)$ of [N7].

3.2. Proof of formulas (2.14), (2.15). We have that

$$
\begin{gathered}
|\hat{v}(k-l)| \stackrel{(3.1),(3.5)}{\leq}(2 \pi)^{-d}\|v\|_{\sigma}\left(c_{1}(d, \sigma)\right)^{2}, \\
\left.|| f(k, l)\right|^{2}-|\hat{v}(k-l)|^{2}|=||f(k, l)|-|\hat{v}(k-l)| \mid(|f(k, l)|+|\hat{v}(k-l)|) \leq \\
|f(k, l)-\hat{v}(k-l)|(2|\hat{v}(k-l)|+|f(k, l)-\hat{v}(k-l)|),
\end{gathered}
$$

where $(k, l) \in \mathcal{M}_{E}, \sigma>d$. Due to (3.4), (3.7), (3.8), we have that

$$
\begin{aligned}
& \left.|| f(k, l)\right|^{2}-|\hat{v}(k-l)|^{2}\left|\leq 3(2 \pi)^{-d}\|v\|_{\sigma}\left(c_{1}(d, \sigma)\right)^{2}\right| f(k, l)-\hat{v}(k-l) \mid \leq \\
& 6(2 \pi)^{-2 d} a_{0}(d, \sigma / 2)\left(\left(c_{1}(d, \sigma)\right)^{4}\left(\|v\|_{\sigma}\right)^{3} E^{-1 / 2},\right.
\end{aligned}
$$


$(k, l) \in \mathcal{M}_{E}, E^{-1 / 2} \geq \rho_{1}\left(d, \sigma,\|v\|_{\sigma}\right), \sigma>d$. Formulas (2.14), (2.15) follow from (3.9) for $v=v_{j}, f=f_{j}$ and from the possibility of choice of $k=k_{E}(p), l=l_{E}(p)$ as in (1.7) for $d \geq 2$. In addition,

$$
\begin{aligned}
& c(D)=6(2 \pi)^{-2 d} a_{0}(d, \sigma / 2)\left(c_{1}(d, \sigma)\right)^{4}\left(c_{2}(d, \sigma)\right)^{3}, \\
& \rho(D, N)=\rho_{1}\left(d, \sigma, c_{2}(D, \sigma) N\right),
\end{aligned}
$$

for some fixed $\sigma>d$, where

$$
c_{2}(D, \sigma)=\sup _{x \in D}\left(1+|x|^{2}\right)^{\sigma / 2}
$$

3.3. Proof of formula (2.20). We have that

$$
\begin{aligned}
& \left|\hat{v}_{1}\right|^{2} \stackrel{(1.17)}{=}\left|\hat{v}+\hat{w}_{1}\right|^{2} \stackrel{(2.3)}{=}|| \hat{v}\left|e^{i \alpha}+\right| \hat{w}_{1}\left|e^{i \beta_{1}}\right|= \\
& \left(|\hat{v}| \cos \alpha+\left|\hat{w}_{1}\right| \cos \beta_{1}\right)^{2}+\left(|\hat{v}| \sin \alpha+\left|\hat{w}_{1}\right| \sin \beta_{1}\right)^{2}= \\
& |\hat{v}|^{2}+\left|\hat{w}_{1}\right|^{2}+2|\hat{v}|\left|\hat{w}_{1}\right|\left(\cos \alpha \cos \beta_{1}+\sin \alpha \sin \beta_{1}\right) \text { on } \mathbb{R}^{d} .
\end{aligned}
$$

Formula (2.20) follows from (3.13).

3.4. Proof of formula (2.17). Using (3.13) and analogous formula for $\hat{v}_{2}=\hat{v}+\hat{w}_{2}$, we obtain the system

$$
\left(\begin{array}{cc}
\cos \beta_{1} & \sin \beta_{1} \\
\cos \beta_{2} & \sin \beta_{2}
\end{array}\right)\left(\begin{array}{c}
\cos \alpha \\
\sin \alpha
\end{array}\right)=\left(\begin{array}{c}
\left(2|\hat{v}|\left|\hat{w}_{1}\right|\right)^{-1}\left(\left|\hat{v}_{1}\right|^{2}-|\hat{v}|^{2}-\left|\hat{w}_{1}\right|^{2}\right) \\
\left(2|\hat{v}|\left|\hat{w}_{2}\right|\right)^{-1}\left(\left|\hat{v}_{2}\right|^{2}-|\hat{v}|^{2}-\left|\hat{w}_{2}\right|^{2}\right)
\end{array}\right)
$$

on $\mathbb{R}^{d} \backslash\left(Z_{0} \cup Z_{1} \cup Z_{2}\right)$.

Formula (2.17) follows from (3.14).

3.5. Final remark. Proposition 2.1 and Theorem 2.1 follow from formulas (2.14), (2.15), (2.20), (2.17) proved in Subsections 3.2, 3.3, 3.4.

\section{Proof of Theorem 2.2}

Let

$$
\Delta_{E_{0}, E}=\left\{(k, l) \in \Gamma_{E}: \quad k-l \in \mathcal{B}_{2 \sqrt{E_{0}}}\right\}, \quad 0<E_{0} \leq E,
$$

where $\Gamma_{E}, \mathcal{B}_{r}$ are defined by (1.7), (1.8).

Theorem 2.2 follows from:

(1) the formulas of Theorem 2.1 with $\Delta_{E_{*}, E}$ in place of $\mathcal{M}_{E}$ and $|p|<2 \sqrt{E_{*}}$,

(2) the fact that $\hat{v}$ on $\mathcal{B}_{2 \sqrt{E_{*}}} \backslash\left(Z_{1} \cup Z_{2} \cup \bar{Y}_{1,2}\right)$ uniquely determines $\hat{v}$ on $\mathbb{R}^{d}$ (since $\hat{v}$ is real-analytic on $\mathbb{R}^{d}$ ), and

(3) the results of Lemma 4.1 for $v$ and for $v=v+w_{j}, j=1,2$.

Lemma 4.1. Let $v$ satisfy (1.2) and be real-valued. Then:

(a) $\left|f\left(k_{E}(p), l_{E}(p)\right)\right|^{2}$ is real-analytic in $\left.E \in\right] p^{2} / 4,+\infty\left[\right.$ for fixed $p \in \mathbb{R}^{d}$, where $k_{E}(p)$, $l_{E}(p)$ are defined in (1.7); 
Explicit formulas and global uniqueness for phaseless inverse scattering in multidimensions

(b) $|f|^{2}$ on $\Gamma_{\Lambda}$ uniquely determines $|f|^{2}$ on $\Delta_{E_{*}, E}$ for each $E \geq E_{*}$, where $\Gamma_{\Lambda}, \Lambda$ are defined in (1.11), (2.19).

Statement (b) of Lemma 4.1 follows from statement (a) of Lemma 4.1 and the property that the accumulation point $\left.E_{*} \in\right] p^{2} / 4,+\infty\left[\right.$ if $p \in \mathcal{B}_{2 \sqrt{E_{*}}}$.

In turn, statement (a) of Lemma 4.1 follows from the presentation

$$
|f|^{2}=f \bar{f}
$$

and from Lemma 4.2 .

Lemma 4.2. Let $v$ satisfy (1.2) and be real-valued. Then $f\left(k_{E}(p), l_{E}(p)\right)$ admits holomorphic extension in $E$ to an open $\mathcal{N}$ in $\mathbb{C}$, where $] p^{2} / 4,+\infty\left[\subset \mathcal{N}\right.$, at fixed $p \in \mathbb{R}^{d}$.

Lemma 4.2 follows from:

(1) the integral equation (1.4) for $\psi^{+}$on $D$ and the presentation (1.5) for $f$, where

$k=k_{E}(p), l=l_{E}(p)$;

(2) the property that

$$
G^{+}(x, k)=G_{0}^{+}(|x|,|k|), \quad x \in \mathbb{R}^{d}, \quad k \in \mathbb{R}^{d} \backslash\{0\},
$$

where $G^{+}$is the function of (1.4) and $G_{0}^{+}$depends also on $d$;

(3) the properties that: $\left|k_{E}(p)\right|=E^{1 / 2}$ for $\left.E \in\right] p^{2} / 4,+\infty\left[, p \in \mathbb{R}^{d} ; E^{1 / 2}\right.$ is holomorphic in $E \in \mathbb{C} \backslash]-\infty, 0] ;\left(E-p^{2} / 4\right)^{1 / 2}$ is holomorphic in $\left.\left.E \in \mathbb{C} \backslash\right]-\infty, p^{2} / 4\right] ; p \in \mathbb{R}^{d}$; $G_{0}^{+}(r, \kappa)$ is holomorphic in $\kappa \in \mathbb{C}$ for odd $d \geq 3$ and in $\left.\kappa \in \mathbb{C} \backslash\right]-\infty, 0$ ] for even $d \geq 2$, where $r>0$;

(4) the result that (1.4) with $k=k_{E}(p)$ is a Fredholm integral equation of the second kind for $\psi^{+}(\cdot, k) \in L^{2}(D)$ with holomorphic dependence on the parameter $\left.E \in \mathbb{C} \backslash]-\infty, p^{2} / 4\right]$ at fixed $p \in \mathbb{R}^{d}$

(5) the result that (1.4) is uniquely solvable for $\psi^{+}(\cdot, k) \in L^{2}(D)$ for each $k \in \mathbb{R}^{d} \backslash\{0\}$ under our assumptions on $v$.

In connection with basic properties of function $G^{+}$and basic properties of the Lippmann-Schwinger integral equation (1.4) we refer also to [BS], [F2], [Me].

\section{Proof of Proposition 2.2}

Proof of part (A). Due to formulas (2.14), (2.15) with $\Gamma_{E}$ in place of $\mathcal{M}_{E}$, we have that $S=\left\{|f|^{2},\left|f_{1}\right|^{2}\right\}$ on $\Gamma_{\Lambda}$ uniquely determine $|\hat{v}|,\left|\hat{v}_{1}\right|$ on $\mathbb{R}^{d}$. If $|\hat{v}| \equiv 0$, then $v=0$ in $L^{\infty}\left(\mathbb{R}^{d}\right)$. Therefore, it remains to consider the case when $|\hat{v}| \not \equiv 0$.

Due to (2.8), (2.9), $j=1$, and continuity of $\hat{v}, \hat{w}_{1}$, we can choose $p^{\prime} \in \mathbb{R}^{d}, r^{\prime}>0$ such that

$$
\begin{aligned}
& |\hat{v}(p)| \neq 0,\left|\hat{w}_{1}(p)\right| \neq 0 \text { for } p \in \mathcal{B}_{p^{\prime}, r^{\prime}}, \\
& \mathcal{B}_{p^{\prime}, r^{\prime}}=\left\{p \in \mathbb{R}^{d}:\left|p-p^{\prime}\right|<r^{\prime}\right\} .
\end{aligned}
$$

Therefore, formula $(2.20)$ for $\cos \left(\alpha-\beta_{1}\right)$ holds for each $p \in \mathcal{B}_{p^{\prime}, r^{\prime}}$.

If $\cos \left(\alpha-\beta_{1}\right) \equiv 1$ on $\mathcal{B}_{p^{\prime}, r^{\prime}}$, then $\alpha \equiv \beta_{1}(\bmod 2 \pi)$ on $\mathcal{B}_{p^{\prime}, r^{\prime}}$. If $\cos \left(\alpha-\beta_{1}\right) \equiv-1$ on $\mathcal{B}_{p^{\prime}, r^{\prime}}$, then $\alpha \equiv \beta_{1}+\pi(\bmod 2 \pi)$ on $\mathcal{B}_{p^{\prime}, r^{\prime}}$. And in both cases $\hat{v}=|\hat{v}| e^{i \alpha}$ is uniquely determined on $\mathcal{B}_{p^{\prime}, r^{\prime}}$ by $|\hat{v}|,\left|\hat{v}_{1}\right|, \hat{w}_{1}=\left|\hat{w}_{1}\right| e^{i \beta_{1}}$ on $\mathcal{B}_{p^{\prime}, r^{\prime}}$. 
Due to continuity of $e^{i \alpha}, e^{i \beta_{1}}$ on $\mathcal{B}_{p^{\prime}, r^{\prime}}$ we can choose $p^{\prime \prime} \in \mathcal{B}_{p^{\prime}, r^{\prime}}$ and $\left.r^{\prime \prime} \in\right] 0, r^{\prime}[$ such that

$$
-1<c_{\min } \leq \cos \left(\alpha-\beta_{1}\right) \leq c_{\max }<1 \text { on } \mathcal{B}_{p^{\prime \prime}, r^{\prime \prime}}
$$

for some fixed $c_{\text {min }}, c_{\max }$. Therefore, due to formula $(2.20)$ for $\cos \left(\alpha-\beta_{1}\right)$ and continuity of $\alpha, \beta_{1}(\bmod 2 \pi)$ on $\mathcal{B}_{p^{\prime \prime}, r^{\prime \prime}}$ we have that either

$$
\alpha=\beta_{1}+\arccos \left(\left(2|\hat{v}|\left|\hat{w}_{1}\right|\right)^{-1}\left(\left|\hat{v}_{1}\right|^{2}-|\hat{v}|^{2}-\left|\hat{w}_{1}\right|^{2}\right)\right)
$$

or

$$
\alpha=\beta_{1}-\arccos \left(\left(2|\hat{v}|\left|\hat{w}_{1}\right|\right)^{-1}\left(\left|\hat{v}_{1}\right|^{2}-|\hat{v}|^{2}-\left|\hat{w}_{1}\right|^{2}\right)\right)
$$

$(\bmod 2 \pi)$ on $\mathcal{B}_{p^{\prime \prime}, r^{\prime \prime}}$, where arc cos takes values in $[0, \pi]$. Therefore, there are not more than two different $\hat{v}=|\hat{v}| e^{i \alpha}$ on $\mathcal{B}_{p^{\prime \prime}, r^{\prime \prime}}$ with given $|\hat{v}|,\left|\hat{v}_{1}\right|, \hat{w}_{1}=\left|\hat{w}_{1}\right| e^{i \beta_{1}}$ on $\mathcal{B}_{p^{\prime \prime}, r^{\prime \prime}}$. In turn, $\hat{v}$ on $\mathcal{B}_{p^{\prime \prime}, r^{\prime \prime}}$ uniquely determines $\hat{v}$ on $\mathbb{R}^{d}$ due to real analyticity of $\hat{v}$.

This completes the proof of part (A) of Proposition 2.2.

Proof of part (B). Due to statement (b) of Lemma 4.1 (for $v$ and for $v=v+w_{1}$ ), $S=\left\{|f|^{2},\left|f_{1}\right|^{2}\right\}$ on $\Gamma_{\Lambda}$ uniquely determine $S$ on $\Delta_{E_{*}, E}$. Due to formulas (2.14), (2.15) with $\Delta_{E_{*}, E}$ in place of $\mathcal{M}_{E}$ and $|p|<2 \sqrt{E_{*}}$, we have that $S$ on $\Delta_{E_{*}, E}$ uniquely determine $|\hat{v}|,\left|\hat{v}_{1}\right|$ on $\mathcal{B}_{2 \sqrt{E_{*}}}$.

Then in a completely similar way with the proof of part (A) of Proposition 2.2 we obtain that there are not more than two different $\hat{v}$ on $\mathcal{B}_{2 \sqrt{E_{*}}}$ with given $|\hat{v}|,\left|\hat{v}_{1}\right|, \hat{w}_{1}$ on $\mathcal{B}_{2 \sqrt{E_{*}}}$.

Finally, $\hat{v}$ on $\mathcal{B}_{2 \sqrt{E_{*}}}$ uniquely determines $\hat{v}$ on $\mathbb{R}^{d}$ due to real analyticity of $\hat{v}$.

This completes the proof of part (B).

\section{References}

[ AS] T. Aktosun, P.E. Sacks, Inverse problem on the line without phase information, Inverse Problems 14, 1998, 211-224.

[ABR] N.V. Alexeenko, V.A. Burov, O.D. Rumyantseva, Solution of the three-dimensional acoustical inverse scattering problem. The modified Novikov algorithm, Acoust. J. 54(3), 2008, 469-482 (in Russian), English transl.: Acoust. Phys. 54(3), 2008, 407-419.

[BS] F.A. Berezin, M.A. Shubin, The Schrödinger Equation, Vol. 66 of Mathematics and Its Applications, Kluwer Academic, Dordrecht, 1991.

[B] A.L. Buckhgeim, Recovering a potential from Cauchy data in the two-dimensional case, J. Inverse Ill-Posed Probl. 16(1), 2008, 19-33.

[BAR] V.A. Burov, N.V. Alekseenko, O.D. Rumyantseva, Multifrequency generalization of the Novikov algorithm for the two-dimensional inverse scattering problem, Acoust. J. 55(6), 2009, 784-798 (in Russian); English transl.: Acoustical Physics 55(6), 2009, $843-856$.

[ChS] K. Chadan, P.C. Sabatier, Inverse Problems in Quantum Scattering Theory, 2nd edn. Springer, Berlin, 1989

[ EW] V.Enss, R.Weder, Inverse potential scattering: a geometrical approach, Mathematical quantum theory. II. Schrödinger operators (Vancouver, BC, 1993), 151-162, CRM Proc. Lecture Notes, 8, Amer.Math.Soc., Providence, RI, 1995. 
Explicit formulas and global uniqueness for phaseless inverse scattering in multidimensions

[E] G. Eskin, Lectures on Linear Partial Differential Equations, Graduate Studies in Mathematics, Vol.123, American Mathematical Society, 2011.

[ER] G. Eskin, J. Ralston, Inverse backscattering problem in three dimensions, Commun. Math. Phys. 124, 1989, 169-215.

[F1] L.D. Faddeev, Uniqueness of the solution of the inverse scattering problem, Vest. Leningrad Univ. 7, 1956, 126-130 [in Russian].

[ F2] L.D. Faddeev, The inverse problem in the quantum theory of scattering.II, Current problems in mathematics, Vol. 3, 1974, pp. 93-180, 259. Akad. Nauk SSSR Vsesojuz. Inst. Naucn. i Tehn. Informacii, Moscow(in Russian); English transl.: J.Sov. Math. 5, 1976, 334-396.

[ G] P.G. Grinevich, The scattering transform for the two-dimensional Schrödinger operator with a potential that decreases at infinity at fixed nonzero energy, Uspekhi Mat. Nauk 55:6(336),2000, 3-70 (Russian); English translation: Russian Math. Surveys 55:6, 2000, 1015-1083.

[ HH] P. Hähner, T. Hohage, New stability estimates for the inverse acoustic inhomogeneous medium problem and applications, SIAM J. Math. Anal., 33(3), 2001, 670-685.

[ HN] G.M. Henkin, R.G. Novikov, The $\bar{\partial}$-equation in the multidimensional inverse scattering problem, Uspekhi Mat. Nauk 42(3), 1987, 93-152 (in Russian); English transl.: Russ. Math. Surv. 42(3), 1987, 109-180.

[ I] M.I. Isaev, Exponential instability in the inverse scattering problem on the energy interval, Funkt. Anal. Prilogen. 47(3), 2013, 28-36 (in Russian); English transl.: Funct. Anal. Appl. 47(3), 2013, 187-194.

[IN] M.I. Isaev, R.G. Novikov, New global stability estimates for monochromatic inverse acoustic scattering, SIAM J. Math. Anal. 45(3), 2013, 1495-1504

[ J] A. Jensen, High energy resolvent estimates for generalized many-body Schrödinger operators, Publ. RIMS Kyoto Univ. 25, 1989, 155-167.

[ K1] M.V. Klibanov, Phaseless inverse scattering problems in three dimensions, SIAM J. Appl. Math. 74, 2014, 392-410.

[ K2] M.V. Klibanov, On the first solution of a long standing problem: uniqueness of the phaseless quantum inverse scattering problem in 3-d, Appl. Math. Lett. 37, 2014, $82-85$.

[LRS] M.M Lavrentev, V.G.Romanov, S.P. Shishatskii, Ill-posed problems of mathematical physics and analysis, Translated from the Russian by J.R.Schulenberger. Translation edited by Levi J.Leifman. Translations of Mathematical Monographs, 64. American Mathematical Society, Providence, RI, 1986.

[ Me] R.B. Melrose, Geometric scattering theory, Cambridge University Press, 1995.

[ Mo] H.E. Moses, Calculation of the scattering potential from reflection coefficients, Phys. Rev. 102, 1956, 559-567.

[ Ne] R.G.Newton, Inverse Schrödinger scattering in three dimensions, Springer, Berlin, 1989.

[N1] R.G. Novikov, Multidimensional inverse spectral problem for the equation $-\Delta \psi+$ $(v(x)-E u(x)) \psi=0$, Funkt. Anal. Prilozhen. 22(4), 1988, 11-22 (in Russian); English transl.: Funct. Anal. Appl. 22, 1988, 263-272. 
[ N2] R.G. Novikov, The inverse scattering problem at fixed energy level for the twodimensional Schrödinger operator, J. Funct. Anal., 103, 1992, 409-463.

[ N3] R.G. Novikov, The inverse scattering problem at fixed energy for Schrödinger equation with an exponentially decreasing potential, Comm. Math. Phys., 161, 1994, 569-595.

[ N4] R.G. Novikov, On determination of the Fourier transform of a potential from the scattering amplitude, Inverse Problems 17, 2001, 1243-1251.

[ N5] R.G. Novikov, The $\bar{\partial}$-approach to monochromatic inverse scattering in three dimensions, J. Geom. Anal. 18, 2008, 612-631.

[ N6] R.G. Novikov, Absence of exponentially localized solitons for the Novikov-Veselov equation at positive energy, Physics Letters A 375, 2011, 1233-1235.

[ N7] R.G. Novikov, An iterative approach to non-overdetermined inverse scattering at fixed energy, Mat. Sb. (to appear)

[ R] T. Regge, Introduction to complex orbital moments, Nuovo Cimento 14, 1959, 951-976

[S] P. Stefanov, Stability of the inverse problem in potential scattering at fixed energy, Annales de l'Institut Fourier, tome 40(4), 1990, 867-884.

[ VW] A.Vasy, X.-P. Wang, Inverse scattering with fixed energy for dilation-analytic potentials, Inverse Problems, 20, 2004, 1349-1354.

[WY] R. Weder, D. Yafaev, On inverse scattering at a fixed energy for potentials with a regular behaviour at infinity, Inverse Problems, 21, 2005, 1937-1952. 